\title{
ON THE SURJECTIVITY OF THE POWER MAPS OF SEMISIMPLE ALGEBRAIC GROUPS
}

\author{
Pralay Chatterjee
}

\begin{abstract}
In this paper we study the surjectivity of the power maps $g \mapsto g^{n}$ for semisimple algebraic groups over an algebraically closed field of arbitrary characteristic. We describe certain necessary and sufficient conditions for surjectivity to hold. The results are applied to characterize the exponentiality of such groups.
\end{abstract}

\section{Introduction}

We fix an algebraically closed field $\mathbb{K}$ and let $p$ denote the the characteristic exponent of $\mathbb{K}$. We recall that $p$ is 1 if the characteristic of $\mathbb{K}$ is zero and the characteristic of $\mathbb{K}$ otherwise. Unless mentioned otherwise all the algebraic groups considered are defined over the field $\mathbb{K}$.

Let $G$ be a connected simple algebraic group. We consider the root system associated to $G$ with respect to some maximal torus of $G$. Let $\Delta$ be the set of simple roots with respect to an order in the root system and let $h=\sum_{\alpha \in \Delta} m_{\alpha} \alpha$ be the highest root. A prime $q$ is said to be a bad prime for the simple group $G$ if $q$ divides $m_{\alpha}$ for some $\alpha \in \Delta$. Now if $G$ is connected semisimple then a prime $q$ is said to be a bad prime for $G$ if $q$ is bad for some simple factor of $G$. A prime is said to be a good prime for $G$ if it is not a bad prime.

Let $n$ be a natural number and $P_{n}: G \rightarrow G$ be the $n$-th power map defined by $P_{n}(g)=g^{n}$ for all $g \in G$. We now state the main theorem.

Theorem A. Let $G$ be a connected semisimple algebraic group. Then $P_{n}: G \rightarrow$ $G$ is surjective if and only if $n$ is prime to $p$, the bad primes for $G$ and the order of the center of $G$. In particular, if $G$ is a connected simple algebraic group then $P_{n}: G \rightarrow G$ is surjective if and only if $n$ is prime to $p$ and one of the following conditions holds (depending on the type of $G$ ).

1. $G$ is of type $A_{l}, l \geq 1$ and $n$ is coprime to the order of the center of $G$.

2. $G$ is of type either $B_{l}, l \geq 2$ or $C_{l}, l \geq 3$ or $D_{l}, l \geq 4$, and $n$ is coprime to 2 and.

3. $G$ is of type either $E_{6}$ or $E_{7}$ or $F_{4}$ or $G_{2}$, and $n$ is coprime to 6 .

4. $G$ is of type $E_{8}$ and $n$ is coprime to 30 .

Hence for any semisimple algebraic group $G$ the map $P_{n}$ is surjective whenever $n$ is coprime to $30 \mathrm{mp}$, where $m$ is the order of its center.

Received February 18, 2003. 
Following M. Moskowitz (cf. [Mo]), we say that an algebraic group $G$ is exponential if every element of $G$ is contained in a connected abelian algebraic subgroup of $G$. From Theorem A we deduce the following criterion for exponentiality of connected semisimple algebraic groups.

Corollary B. Let $G$ be a connected semisimple algebraic group. Then the following are equivalent.

1. $G$ is exponential.

2. $P_{n}: G \rightarrow G$ is surjective for all $n$ prime to $p$ and $p$ is a good prime for $G$ if $p>1$.

3. $G$ is isogenous to a product of groups of type $A_{l}, l \geq 1$ and the reduced center of $G$ is trivial.

The author had earlier proved results characterizing surjectivity of the $n$-th power maps and exponentiality of connected algebraic groups (not necessarily semisimple) over algebraically closed fields of characteristic zero (see Theorem A, Theorem 4.1, Corollary D and Corollary 6.2 of [Ch]). As a consequence a result similar to Theorem $\mathrm{A}$ is proved for semisimple algebraic groups over algebraically closed fields of characteristic zero (see Theorem $\mathrm{C}$ of $[\mathrm{Ch}]$ ). It may be mentioned that the overall approach followed in the proof of Theorem A is similar to that of the proof of Theorem $\mathrm{C}$ in [Ch]. Various steps however call for some essential modifications in the case of arbitrary characteristic.

Prefatory Note: After proving the analogous results in $[\mathrm{Ch}]$ for characteristic zero I had been thinking in particular about the general case. Before the work as presented here in this respect was completed, I was informed by Prof. A. Borel that Prof. R. Steinberg had generalized the results of [Ch] to arbitrary characteristic. The first version of the present manuscript was completed shortly thereafter, without access to Prof. Steinberg's arguments (see [St]). Subsequent comparison shows that the present proof involves substantially different arguments than [St], and may be of independent interest.

\section{Preliminaries}

We recall some basic facts that are needed in the proof of Theorem A. The reader is referred to [B] for generalities in the theory of algebraic groups. Let $G$ be an algebraic group. For any element $x \in G$ the semisimple and the unipotent Jordan components of $x$ will be denoted by $x_{s}$ and $x_{u}$ respectively; then we have $x=x_{s} x_{u}=x_{u} x_{s}$. For an element $g \in G$ let $\overline{\langle g\rangle}$ denote the Zariski closure of the group generated by $g$.

Theorem 2.1. Let $G$ be a connected reductive algebraic group. Then $G$ admits a unipotent element $u$ such that $u$ is contained in a unique maximal unipotent subgroup $U$. For any such $u, Z_{G}(u)=Z_{U}(u) Z(G)$. In particular, the semisimple elements in $Z_{G}(u)$ are central in $G$. 
Let $T$ be a maximal torus in $G$. Consider an ordering in the root system of $G$ with respect to $T$. Let $U$ be the unipotent radical of the Borel subgroup associated to the set of positive roots. One can obtain such a unipotent element $u \in G$ by taking $u$ to be any element in $U$ whose components for the simple roots are all nontrivial. See $[\mathrm{Hu}]$, Sections 4.1-4.5 for details.

We also need the following result.

Theorem 2.2. Let $G$ be a connected semisimple algebraic group and $s \in G$ be a semisimple element. Then $Z_{G}(s)^{0}$ is reductive and all unipotent elements of $Z_{G}(s)$ lie in $Z_{G}(s)^{0}$.

See $[\mathrm{Hu}]$, Section 2.2 for a proof of Theorem 2.2.

We now collect a preliminary result which will be used later.

Lemma 2.3. Let $G$ be an algebraic group and $p>1$. Then there exists a positive integer $k_{G}$ such that for any unipotent element $u \in G$ we have $u^{p^{k_{G}}}=e$. In particular, if $G$ admits a nontrivial unipotent element then $P_{p}: G \rightarrow G$ is not surjective.

Proof. We first embed $G$ in $G L_{d}(\mathbb{K})$ for some integer $d$. We next note that for any unipotent matrix $U$ in $G L_{d}(\mathbb{K})$ we have $U^{p^{d}}=\mathrm{Id}$. The proof is now clear.

Corollary 2.4. Let $G$ be an algebraic group. Let $u \in G$ be an unipotent element. If $n$ is prime to $p$ then there exists $v \in \overline{\langle u\rangle}$ such that $u=v^{n}$.

Proof. If $p=1$ and $u$ is different from identity then $\overline{\langle u\rangle}$ is one dimensional connected unipotent group. Hence the proof is clear in this case. If $p>1$ the proof follows from the lemma above.

\section{Characterisation of the surjectivity of $P_{n}$}

In this section we prove Theorem A. To prove Theorem A we need the following result.

Theorem 3.1. Let $G$ be a connected semisimple algebraic group. Then $P_{n}$ : $G \rightarrow G$ is surjective if and only if $n$ is prime to $p$ and to the order of the finite group $Z_{G}(u) / Z_{G}(u)^{0}$, for all unipotent elements $u \in G$.

We first prove the following lemmas, in preparation of the proof of Theorem 3.1 .

Definition: Let $G$ be a connected algebraic group and $s \in G$ be a semisimple element. A semisimple element $t \in G$ is said to be a $P_{n}$-regular $n$-th root of $s$ if $t^{n}=s$ and $t \in Z\left(Z_{G}(s)^{0}\right)$.

Lemma 3.2. Let $G$ be a connected semisimple algebraic group. Then $P_{n}: G \rightarrow$ $G$ is surjective if and only if $n$ is prime to $p$ and every semisimple element in $G$ has a $P_{n}$-regular $n$-th root. 
Proof. Assume that $P_{n}: G \rightarrow G$ is surjective. By Lemma 2.3 it follows that $n$ is coprime to the prime $p$. Let $s \in G$ be a semisimple element. Note that the group $H=Z_{G}(s)^{0}$ is reductive (cf. Theorem 2.2) and hence $H$ contains regular unipotent elements. Let $u$ be a regular unipotent element in $H$. As $P_{n}$ is surjective there exists $g \in G$ such that $s u=g^{n}$. Note that $g_{s}^{n}=s$ and hence $g_{s} \in H$. We also have $g_{u}^{n}=u$ and hence $g_{s} \in Z_{H}(u)$. We now apply Theorem 2.1 to conclude that $g_{s} \in Z(H)$. Thus $g_{s}$ is a $P_{n}$-regular $n$-th root of $s$.

We now prove the converse. We assume that $n$ is prime to $p$ and that every semisimple element in $G$ has a $P_{n}$-regular $n$-th root. Let $g \in G$. Let $h$ be a $P_{n}$-regular $n$-th root of $g_{s}$. Hence $h^{n}=g_{s}$ and $h \in Z\left(Z_{G}\left(g_{s}\right)^{0}\right)$. Note that $g_{u} \in Z_{G}\left(g_{s}\right)^{0}$ (cf. Theorem 2.2) and hence $h$ commutes with $g_{u}$. As $n$ is prime to $p$, by Corollary 2.4, there exists an element $v \in \overline{\left\langle g_{u}\right\rangle}$ such that $g_{u}=v^{n}$. It is clear that $h$ and $v$ commute. Hence $g=(h v)^{n}$. Thus $P_{n}: G \rightarrow G$ is surjective.

Lemma 3.3. Let $G$ be a connected semisimple algebraic group and let $P_{n}: G \rightarrow$ $G$ be surjective. Then $P_{n}: Z_{G}(u) \rightarrow Z_{G}(u)$ is surjective for every unipotent element $u \in G$.

Proof. Applying Lemma 2.3 we first note that $n$ must be prime to $p$. Let $u$ be an unipotent element in $G$ and $g \in Z_{G}(u)$. Note that $Z_{G}\left(g_{s}\right)^{0}$ is reductive and both $u$ and $g_{u}$ lie in $Z_{G}\left(g_{s}\right)^{0}$ (cf. Theorem 2.2). As $P_{n}: G \rightarrow G$ is surjective, by Lemma 3.2 it follows that $g_{s}$ has a $P_{n}$-regular $n$-th root, say $t$. In other words, $t^{n}=g_{s}$ and $t \in Z\left(Z_{G}\left(g_{s}\right)^{0}\right)$. As $n$ is coprime to $p$, by Corollary 2.4 there exists $v \in \overline{\left\langle g_{u}\right\rangle}$ such that $g_{u}=v^{n}$. Hence $g=(t v)^{n}$. Clearly $t v \in Z_{G}(u)$.

Corollary 3.4. Let $G$ be a connected semisimple algebraic group. such that $P_{n}: G \rightarrow G$ is surjective. Then $P_{n}: Z(G) \rightarrow Z(G)$ is surjective.

Proof. Let $u$ be a unipotent element in $G$ for which the contention of Theorem 2.1 holds. By Lemma 3.3, we have that $P_{n}: Z_{G}(u) \rightarrow Z_{G}(u)$ is surjective. Let $U$ be the maximal unipotent group containing $u$. Note that $Z_{G}(u)$ is a direct product of the groups $Z(G)$ and $Z_{U}(u)$ (cf. Theorem 2.1). Hence $P_{n}: Z(G) \rightarrow Z(G)$ is surjective.

Lemma 3.5. Let $G$ be a connected semisimple algebraic group and $s \in G$ be a semisimple element. Let $m$ and $n$ be coprime integers. If $s^{m}$ has a $P_{n}$-regular $n$-th root then so does $s$.

Proof. Let $t \in G$ be a $P_{n}$-regular $n$-th root of $s^{m}$. First observe that $s \in$ $Z_{G}\left(s^{m}\right)^{0}$. Now as $t \in Z\left(Z_{G}\left(s^{m}\right)^{0}\right)$ we have that $t$ and $s$ commute. As $m$ and $n$ are coprime there exists integers $a, b$ such that $a m+b n=1$. Since $s$ and $t$ commute, we have that $\left(s^{b} t^{a}\right)^{n}=s^{b n} t^{a n}=s^{a m+b n}=s$; that is, $s^{b} t^{a}$ is a $n$-th 
root of $s$. We next show that $s^{b} t^{a} \in Z\left(Z_{G}(s)^{0}\right)$. This will prove that $s^{b} t^{a}$ is a $P_{n}$-regular $n$-th root of $s$. Observe that $s^{b} t^{a} \in Z_{G}(s)^{0}$. Let $x \in Z_{G}(s)^{0}$. Clearly, $x \in Z_{G}\left(s^{m}\right)^{0}$. As $t$ is central in $Z_{G}\left(s^{m}\right)^{0}$ it follows that $x$ and $t$ commutes. Thus $x$ commutes with $s^{b} t^{a}$. Hence $s^{b} t^{a} \in Z\left(Z_{G}(s)^{0}\right)$.

Proof of Theorem 3.1. Suppose $P_{n}: G \rightarrow G$ is surjective. Clearly $n$ is prime to $p$ (cf. Lemma 2.3). Let $u \in G$ be any unipotent element. Then by Lemma 3.3 it follows that $P_{n}: Z_{G}(u) \rightarrow Z_{G}(u)$ is surjective, and hence the induced map $P_{n}: Z_{G}(u) / Z_{G}(u)^{0} \rightarrow Z_{G}(u) / Z_{G}(u)^{0}$ is surjective. As $Z_{G}(u) / Z_{G}(u)^{0}$ is a finite group this implies that its order is prime to $n$.

To prove the converse we first note that $P_{n}$ is surjective if and only if $P_{q}$ is surjective for all prime divisors $q$ of $n$, and hence we may assume $n$ itself to be a prime. Now suppose that $n$ is a prime different from $p$ and that $P_{n}: G \rightarrow G$ is not surjective. Then by Lemma 3.2 there exists a semisimple element which has no $P_{n}$-regular $n$-th root. Let $s$ be such an element for which, furthermore, $Z_{G}(s)$ is of maximum possible dimension. Let $M=Z_{G}(s)^{0}$. Clearly $s \in Z(M)$. As $s$ has no $P_{n}$-regular $n$-th root it follows that $s$ has no $n$-th root in $Z(M)$. Since $Z(M)^{0}$ is a torus of the reductive group $M$, it follows that the element $s Z(M)^{0}$ has no $n$-th root in the quotient group $Z(M) / Z(M)^{0}$. Hence $n$, which is a prime, must divide the order of $s Z(M)^{0}$ in $Z(M) / Z(M)^{0}$.

Let ord(.) denote the order of an element in a group. Now ord $\left(s Z(M)^{0}\right)$ can be expressed as $n^{k} m$ where $k \geq 1$ and $m$ is prime to $n$. Let $\tilde{u}$ be a unipotent element of $M$ for which the contention of Theorem 2.1 holds. Clearly, $s \in Z_{G}(\tilde{u})$. We claim that $n$ divides the order of the element $s Z_{G}(\tilde{u})^{0}$ in the quotient group $Z_{G}(\tilde{u}) / Z_{G}(\tilde{u})^{0}$. If possible suppose that $n$ is prime to $\operatorname{ord}\left(s Z_{G}(\tilde{u})^{0}\right)$. Since $s^{n^{k} m} \in Z(M)^{0}$, it follows that $s^{n^{k} m} \in Z_{G}(\tilde{u})^{0}$. Thus ord $\left(s Z_{G}(\tilde{u})^{0}\right)$ divides $n^{k} m$. As $n$ is prime to $\operatorname{ord}\left(s Z_{G}(\tilde{u})^{0}\right)$ we conclude that $\operatorname{ord}\left(s Z_{G}(\tilde{u})^{0}\right)$ divides $m$. Thus $s^{m} \in Z_{G}(\tilde{u})^{0}$. Let $T$ be torus in $Z_{G}(\tilde{u})^{0}$ such that $s^{m} \in T$. Clearly, $T \subset Z_{G}\left(s^{m}\right)^{0}$. Now as $m$ is prime to $n$, by Lemma 3.5 it follows that $s^{m}$ also has no $P_{n}$-regular $n$-th root in $G$. Thus by maximality of the dimension of $Z_{G}(s)$ it follows that $M=Z_{G}\left(s^{m}\right)^{0}$. Thus $T \subset M$ and hence $T \subset Z_{M}(\tilde{u})$. As $T$ is a connected group consisting of semisimple elements, by Theorem 2.1 it follows that $T \subset Z(M)^{0}$. Hence $s^{m} \in Z(M)^{0}$. Thus $\operatorname{ord}\left(s Z(M)^{0}\right)$ divides $m$. This is a contradiction since $n$ divides ord $\left(s Z(M)^{0}\right)$ and $m$ is prime to $n$. Thus $n$ divides the order of the element $s Z_{G}(\tilde{u})^{0}$ in the quotient group $Z_{G}(\tilde{u}) / Z_{G}(\tilde{u})^{0}$. Hence the order of the quotient group $Z_{G}(\tilde{u}) / Z_{G}(\tilde{u})^{0}$ is divisible by $n$. This completes the proof.

We shall next apply Theorem 3.1 to prove Theorem A. We also need the following lemma which is a special case of a result due to T.A. Springer and R. Steinberg. 
Lemma 3.6. Let $G$ be a connected simple adjoint group and let $u \in G$ be an unipotent element. Then any prime divisor of the order of $Z_{G}(u) / Z_{G}(u)^{0}$ is either $p$ or a bad prime for $G$.

Proof. Note that for any unipotent element $\tilde{u} \in Z_{G}(u)$ there exists a positive integer $r$ such that $\tilde{u}^{p^{r}} \in Z_{G}(u)^{0}$. Combining this fact and Theorem 3.17, Section III of [S-St] we get the proof.

We next note a converse of Lemma 3.6.

Lemma 3.7. Let $G$ be a connected simple adjoint group and $q$ be a bad prime for $G$. Assume further that $q$ is different from $p$. Then there exists a unipotent element $u \in G$ such that $q$ divides the order of $Z_{G}(u) / Z_{G}(u)^{0}$.

Proof. Let $T$ be a maximal torus of $G$. We consider the root system associated to $G$ with respect to $T$. Let $\Delta$ be the set of simple roots with respect to an order in the root system. Let us denote the highest root $\sum_{\alpha \in \Delta} m_{\alpha} \alpha$ by $\lambda$. Let $q$ be any bad prime, so that $q$ divides some coefficient, $m_{\alpha_{0}}$ say, of the highest root $\lambda=\sum_{\alpha \in \Delta} m_{\alpha} \alpha$. As $G$ is adjoint there exists a unique $t \in T$ such that $\alpha_{0}(t)=\omega$, a primitive $q$-th root of 1 , and $\alpha(t)=1$ for every other simple root $\alpha$. Clearly the order of $t$ is $q$. We denote the group $Z_{G}(t)^{0}$ by $H$. Note that $H$ is a reductive subgroup of $G$ containing $T$. We observe that the set $\{\lambda\} \cup\left\{\alpha \in \Delta \mid \alpha \neq \alpha_{0}\right\}$ of roots of $G$ will also be a set of roots of $H$ with respect to the maximal torus $T$. This implies that $Z(H)$ is finite (and hence $H$ is semisimple). Let $u$ be a unipotent element of $H$ for which the contention of Theorem 2.1 holds. We claim $t \notin Z_{G}(u)^{0}$. If not, then $t \in T_{1}$ for some torus $T_{1}$ in $Z_{G}(u)^{0}$. Clearly, $u$ commutes element wise with $T_{1}$. However, in the semisimple group $H$ only finitely many semisimple elements can commute with the unipotent element $u$, viz., those of the center (cf. Theorem 2.1 ). This contradiction establishes the claim. Hence $q$ divides the order of $Z_{G}(u) / Z_{G}(u)^{0}$.

For a connected simple algebraic group $G$ let $\mathcal{B}(G)$ denote the set of bad primes for $G$. The relevant facts on $\mathcal{B}(G)$ and order of centers of $G$ for connected simple algebraic groups may be summarized as follows (see Theorem 4.3, Section I of [S-St] and Section 1.11 of $[\mathrm{C}]$ )

Proposition 3.8. Let $G$ be a simple algebraic group and $m$ be the order of the center of $G$. Then we have the following.

1. $\mathcal{B}(G)=\emptyset$ and $m$ divides $l+1$ if $G$ is of type $A_{l}, l \geq 1$.

2. $\mathcal{B}(G)=\{2\}$ and $m$ divides 4 , if $G$ is of type $B_{l}, l \geq 2$, or $C_{l}, l \geq 3$ or $D_{l}, l \geq 4$.

3. $\mathcal{B}(G)=\{2,3\}$ and $m$ divides 6 if $G$ is of type $E_{6}, E_{7}, F_{4}$ or $G_{2}$.

4. $\mathcal{B}(G)=\{2,3,5\}$ and $m=1$ if $G$ is of type $E_{8}$. 
Proof of Theorem A. By Corollary $3.4 P_{n}: G \rightarrow G$ is surjective if and only if $P_{n}: Z(G) \rightarrow Z(G)$ and $P_{n}: G^{\prime} \rightarrow G^{\prime}$ are surjective, where $G^{\prime}=G / Z(G)$. Since $P_{n}: Z(G) \rightarrow Z(G)$ is surjective if and only if $n$ is prime to the order of $Z(G)$ to prove Theorem A it suffices to show that if $G$ has trivial center then $P_{n}$ is surjective if and only if $n$ is prime to the bad primes for $G$ and $p$. When $G$ has trivial center it is a direct product $G=\prod_{l} G_{l}$, where $G_{i}, i=1, \ldots, l$ are connected center-free simple algebraic groups. A prime is bad for $G$ if and only if it is bad for some $G_{i}$ and $P_{n}$ is surjective for $G$ if and only if it is surjective for each $G_{i}$. Therefore it suffices to prove the theorem for simple algebraic groups with trivial center and for this case we apply Theorem 3.1, Lemma 3.6 and Lemma 3.7 to conclude the proof. This completes the proof of the first part of Theorem A. The proof of the latter parts follow immediately from Proposition 3.8 .

\section{Application to exponentiality}

In this section we apply Theorem A and Theorem 3.1 to prove Corollary B.

For an algebraic group $G$ we denote the variety of unipotent elements of $G$ by $\mathcal{U}_{G}$ and the variety of nilpotent elements of the Lie algebra of $G$ by $\mathcal{N}_{G}$. We need the following result due to T.A. Springer.

Theorem 4.1. Suppose $G$ is a simply connected semisimple algebraic group and $p$ is a good prime for $G$. Then there exists a $G$-equivariant isomorphism from $\mathcal{N}_{G}$ to $\mathcal{U}_{G}$.

For a proof of Theorem 4.1 see Theorem 3.1 of $[\mathrm{S}]$.

Corollary 4.2. Suppose $G$ is a connected reductive algebraic group and $p$ is a good prime for $G$. Then any unipotent element of $G$ lies in a connected abelian closed unipotent subgroup of $G$.

Proof. First observe that it is enough to prove the corollary for $G$ semisimple. We next note that a central isogeny between two semisimple groups $H$ and $\widetilde{H}$ induces isomorphisms of $\mathcal{N}_{H}$ onto $\mathcal{N}_{H}$ and of $\mathcal{U}_{H}$ onto $\mathcal{U}_{H}$. This allows us to see that Theorem 4.1 is valid for any connected semisimple algebraic group. Thus, as $p$ is good for $G$, there exists a $G$-equivariant isomorphism $f: \mathcal{N}_{G} \rightarrow \mathcal{U}_{G}$. Let

$u \in G$ be unipotent. We choose $X \in \mathcal{N}_{G}$ such that $u=f(X)$. Let $S$ be the set of elements $f(t X), t \in \mathbb{K}$. Clearly $S$ is a closed connected set containing the identity of $G$. As $f$ is a $G$-equivariant isomorphism it follows that $S$ is a commutative set of unipotent elements. So the group generated by $S$ is connected, abelian, unipotent and contains $u$. 
Remark 4.3. Here we mention a result similar to Corollary 4.2, but stronger if we impose a condition on the unipotent elements. It is proved by D. Testerman (see Theorem 0.1, [T]) that if $G$ is a semisimple group and $p$ is a good prime for $G$ then any unipotent element $u$ of order $p$ lies in a closed subgroup $H$ of $G$, with $H$ being isomorphic to $S L_{2}(\mathbb{K})$ or $P S L_{2}(\mathbb{K})$.

We also need the following result due to T.A. Springer and R. Steinberg.

Theorem 4.4. Suppose $G$ is a semisimple algebraic group and $p$ is a bad prime for $G$. Then there exists a unipotent element $u \in G$ such that $u \notin Z_{G}(u)^{0}$.

See 1.14 (d) in III of [S-St] for a proof of Theorem 4.4.

We now give two proofs of Corollary B. The first one uses Corollary 4.2 and the second one does not.

Proof of Corollary B.

Proof 1: We first prove $(1 \Rightarrow 2)$. As $G$ is exponential every element lies in a connected abelian closed subgroup. For a connected abelian group $H$, the power map $P_{n}: H \rightarrow H$ is surjective if $n$ is prime to $p$. To complete the proof of the above implication we now assume $p>1$ and show that $p$ is good for $G$. If $p$ is bad then by Theorem 4.4 there exists an unipotent element $u$ such that $u \notin Z_{G}(u)^{0}$. Now as $G$ is exponential $u$ will lie in a connected unipotent abelian subgroup. Thus $u \in Z_{G}(u)^{0}$ and we arrive at a contradiction. Hence if $p>1$ then $p$ is good for $G$. We now prove $(2 \Rightarrow 1)$. Let $g \in G$. First assume that $p>1$. As $P_{n}$ is surjective for all $n$ prime to $p$, it follows from Theorem 3.1 that the order of the finite group $Z_{G}\left(g_{u}\right) / Z_{G}\left(g_{u}\right)^{0}$ is some power of $p$. Hence $g_{s} \in Z_{G}\left(g_{u}\right)^{0}$. Let $T$ be a torus in $Z_{G}\left(g_{u}\right)^{0}$ containing $g_{s}$. As $p$ is good for $G$ and as $Z_{G}(T)$ is a Levi subgroup of some parabolic subgroup of $G$ it follows that $p$ is good for $Z_{G}(T)$. Clearly $g_{u} \in Z_{G}(T)$. Now by Corollary $4.2, g_{u}$ is contained in a connected abelian unipotent subgroup $U$ of $Z_{G}(T)$. Then $T U$ is a connected abelian subgroup of $G$ containing $g$. Now if $p=1$ then it follows from Theorem 3.1 that $Z_{G}\left(g_{u}\right)=Z_{G}\left(g_{u}\right)^{0}$. Let $T$ be a torus in $Z_{G}\left(g_{u}\right)$ containing $g_{s}$. We note that $g$ is contained in the connected abelian group $T \overline{\left\langle g_{u}\right\rangle}$.

We now prove the equivalence of 2 and 3. If $P_{n}: G \rightarrow G$ is surjective for all $n$ prime to $p$ then from Corollary 3.4 it follows that the order of $Z(G)$ is a power of $p$. The proof is now clear from Theorem A.

Proof 2: We now give the second proof of Corollary B. The implications $(1 \Rightarrow 2)$ and $(2 \Rightarrow 3)$ are established as before. We now show $(3 \Rightarrow 1)$. It suffices to show that any element $g$ in $G L_{n}(\mathbb{K})$ lies in a connected abelian closed subgroup. Let $A$ be the $\mathbb{K}$-subalgebra generated by $g$ in the algebra of $n \times n$ matrices $M_{n}(\mathbb{K})$. We observe that the intersection of $A$ and $G L_{n}(\mathbb{K})$ is a closed, abelian subgroup of $G L_{n}(\mathbb{K})$ which is also a irreducible subvariety containing $g$. This completes the proof. 


\section{Acknowledgements}

I would like to thank Prof. A. Borel for his comments on an earlier version of

this paper. I would also like to thank Prof. Gopal Prasad and Prof. R. Steinberg for suggesting the present proofs of Corollary B. Thanks are also due to Prof. S.G. Dani for helpful suggestions.

\section{References}

[B] A. Borel, Linear Algebraic Groups, Second Edition, GTM-126, Springer-Verlag, 1991.

[C] R. W. Carter, Finite Groups of Lie Type: Conjugacy Classes and Complex Characters, Wiley-Interscience, 1985.

[Ch] P. Chatterjee, On the surjectivity of the power maps of algebraic groups in characteristic zero, Math. Res. Letters, 9, (2002), 741-756.

[Hu] J. E. Humphreys, Conjugacy Classes in Semisimple Algebraic Groups, Math. Surveys and Monographs, 43, AMS, 1995.

[Mo] M. Moskowitz, Exponentiality of algebraic groups, J. Algebra 186, (1996), 20-31.

[S] T. A. Springer The unipotent variety of a semisimple group, Algebraic Geometry, International Colloquium, Tata Institute of Fundamental Research, Bombay, 1968, Oxford University Press, (1969), 373-391.

[St] R. Steinberg, On power maps in algebraic groups, (preprint).

[S-St] T. A. Springer and R. Steinberg, Conjugacy classes, Seminar on Algebraic Groups and Related Finite Groups, The Institute for Advanced Study, Princeton, 1968/69, Lecture Notes in Math. 131, Springer-Verlag, (1970), 167-266.

[T] D.M. Testerman, A1-type overgroups of elements of order $p$ in semisimple algebraic groups and the associated finite groups, J. Algebra 177, (1995), 34-76.

Department of Mathematics, Oklahoma State University, Stillwater, OK 74078, USA.

E-mail address: cpralay@math.okstate.edu

Current Address: Department of Mathematics, Rice University, 6100 Main Street, Houston, TX 77005, USA.

E-mail address: pralay@rice.edu 